\title{
Atypical antipsychotic Lumateperone Beyond Schizophrenia: Seeking clarity in a time of Uncertainty
}

\section{Wael MY Mohamed ${ }^{1,2}$}

${ }^{1}$ Clinical pharmacology department, Menoufia Medical School, Menoufia University, Egypt

${ }^{2}$ Basic Medical Science Department, Kulliyyah of Medicine, International Islamic University Malaysia (IIUM), Pahang, Malaysia

\section{*Corresponding author:}

Wael Mohamed

wmy107@gmail.com

\begin{abstract}
:
Lumateperone (ITI-007) is a serotonin 5HT2A tosylate salt with high affinity for dopamine D2 and D1 receptors and the serotonin transporter. It is unusual in that it controls serotonin, dopamine, and glutamate neurotransmission concurrently, all of which have been implicated in severe mental illness. Consider it a multi-targeted ligand and multifunctional modulator of the serotoninergic system with possible precognitive, antipsychotic, antidepressant, and anxiolytic properties. While lumateperone has been explored as a new agent for schizophrenia therapy, it also provides a unique therapeutic option for a range of other psychiatric and neurological diseases, including behavioural signs of dementia or Alzheimer's disease, sleep problems, and bipolar depression. Additionally, it had a better safety profile than placebo, with no significant extrapyramidal side effects, hyperprolactinemia, or changes in cardiometabolic or endocrine characteristics. Additional study is needed to validate and analyse lumateperone's
\end{abstract}


effectiveness, as well as to identify prospective therapeutic targets. This article gives a comprehensive overview of the most notable results and potential future applications of this chemical in personalised medicine, particularly for neurodegenerative diseases.

Key words: Atypical antipsychotics; Lumateperone; Neurodegenerative, AD, PD

\section{1- Introduction and Background:}

Schizophrenia care improved considerably, in large part due to the advent of the secondgeneration antipsychotics. Schizophrenia patients may now have an expanded range of treatment options because of the discovery that certain 5HT-2A antagonists can reduce the adverse motor consequences of D2 receptor blockade. However, antipsychotics have historically been more successful against positive symptoms (symptoms indicative of wellbeing, such as creativity and pleasant emotions) than negative symptoms (symptoms indicative of suffering, such as emotions of anxiety and depression). There is a greater

possibility of developing metabolic issues such as obesity and hyperprolactinemia due to this additional factor (Kantrowitz, 2020). Such a medication would be very advantageous, as antipsychotics with reduced side effects and greater versatility for the treatment of schizophrenia are currently unavailable (Vyas et al., 2020). Lumateperone is an example of a medicine in this class. An antipsychotic drug with a novel mechanism of action, which is suitable for the treatment of schizophrenia, was recently authorised by the FDA (Blair, 2020). There are also some indications that placebo-like metabolic side effects are quite common, a very low incidence of extrapyramidal symptoms, and that the clinical trials of schizophrenia more comprehensively encompass its distressing symptoms (Kantrowitz et al., 2020; Corponi et al., 2019). Research is being done to investigate the long-term safety and effectiveness of the therapy in the case of acute schizophrenia (Kantrowitz et al., 2020; 
Calsolaro et al., 2019). Furthermore, lumateperone is being studied in clinical trials for bipolar depressive disorder and agitation in the setting of Alzheimer disease and other dementias (Kantrowitz et al., 2020; Corponi et al., 2019; Vanover et al., 2019; Correll et al., 2020; Ahmed et al., 2019). Four-week, double-blind, placebo-controlled Phase II and Phase III trials, as well as a six-week, double-blind, placebo-controlled Phase III study, confirmed the efficacy of lumateperone in treating acute exacerbation of schizophrenia at a dosage of $42 \mathrm{mg} / \mathrm{day}$. Additionally, some data points to the notion that people who are doing well on their current antipsychotic medication may see improvements in their condition if they were to take lumateperone as an adjuvant therapy (Corponi et al., 2019; Krogmann et al., 2019). A double-blind, placebo-controlled study found that Lumateperone had a beneficial effect on the symptoms of bipolar depression in one trial, but no significant effect in the other (Mazza et al., 2020). When researchers discovered that the project was not likely to help dementia patients in the long run, the trial was discontinued (Greenwood et al., 2021).

\section{2- Dynamic and Kinetic of Lumateperone}

Lumateperone (Caplyta $\left.{ }^{\circledR}\right)$ is a novel FDA-approved medication used for the treatment of schizophrenia on a mechanistic basis (Blair, 2020). The new study is assessing whether it may also help treat both bipolar depression and behavioural agitation that are commonly associated with Alzheimer's disease (Kantrowitz, 2020; Correll et al., 2020). This drug is useful for treating positive and negative symptoms, as well as cognitive impairment, in schizophrenia (Edinoff et al., 2020). It is an antagonist of the serotonin 5-HT2A receptor, partial agonist of the presynaptic dopamine D2 receptor, and postsynaptic antagonist (Snyder et al., 2015). (Correll et al., 2020). It has little or no antimuscarinic or 
antihistaminic action while yet displaying antagonistic activity against alpha-1 receptors (Caplyta, 2018). Lumateperone is far less likely to cause additional problems than many other antipsychotic medicines, such sleepiness, fatigue, drowsiness when sleeping, constipation, and dry mouth (Correll et al., 2020). The FDA raised concern regarding pigment deposit formation connected to Lumateperone accumulation in several tissues of animal subjects (Caplyta, 2018). It affects and regulates glutaminergic neurotransmission by interacting with and regulating dopaminergic, serotonergic, and NMDA receptors (Corponi et al., 2019; Correll et al., 2020). Unlike the other dopamine agonists, which operate on both pre- and postsynaptic dopamine receptors, this compound exclusively acts on $\mathrm{D} 2$ receptors, where it displays pre- and postsynaptic actions. The molecule works as a D2 dopamine receptor partial agonist and antagonist (Vanover et al., 2019). Additionally, the D2 receptor activity in the mesocortical and mesolimbic circuits is circuit-specific, while there is no evidence of D2 receptor activity in the nigrostriatal dopamine pathways (Kantrowitz et al., 2020; Vyas et al., 2020; Vanover et al., 2019; Krogmann et al., 2019). Gsk-3 phosphorylation results from post-synaptic Lumateperone inhibition of D2 receptors. In the prefrontal cortex and nucleus accumbens, GSK3 is found in neurons that express D2R (Vyas et al., 2020). It is 60 times more specific than D2 receptors (Kantrowitz et al., 2020). A dose-dependent efficacy profile, as well as a tolerability profile that is typically favourable, are common in Lumateperone. These two features may have originated from D2 receptor activity, which is specifically and regionally synaptic and also from the D2:5-HT2A receptor affinity ratio of 60:1. (Kantrowitz et al., 2020; Vancover et al., 2019). while possessing D2 receptor occupancy levels of 40\%, lumateperone has the capability of possessing antipsychotic efficacy while being as low as $40 \%$ in D2 receptor 
occupancy, another common trait found in antipsychotics. It is additionally notable that lumateperone's binding affinity for 5-HT2A receptors is comparable to D2 receptors, which may reduce the occurrence of hyperprolactinemia and extrapyramidal side effects in clinical trials (Vanover et al. 2019). Additional benefits are antidepressant, antianxiety, and antipsychotic effects, and SERT inhibition is involved, making this substance an antidepressant and antipsychotic agent for people with schizophrenia (Vyas et al., 2020). Lumateperone is one of a kind, in that it works on glutaminergic signalling by acting on other signalling pathways. D1 receptor signalling may have phosphorylated the Glu N2B receptor, which was a result of this (Vanover et al., 2019). It is uncertain if lumateperone enhances glutamate signalling via both AMPA and NMDA receptors, although it appears to do so based on the fact that the extent of glutamate activation is greater. In schizophrenia, NMDA receptor activity is known to be low, therefore this effect may be crucial in the drug's antipsychotic and antidepressant characteristics (Vyas et al., 2020; Kumar, 2020). Lumateperone has a dose-dependent clinical impact profile, indicating the drug may have a wider therapeutic range (Vyas et al., 2020). Lumateperone possesses sedative and anti-aggressive effects when used at low doses. Due to the absence of D2 receptor binding and strong selective 5HT2A antagonist activity at low doses, Lumateperone may be able to achieve these effects at low doses. To give more D2 receptors and occupancy and boost the affinity of the 5HT2A receptors, as well as 5HT2A receptor occupancy, one need to raise the doses (Vyas et al., 2020). It is taken orally in capsule form at a prescribed dose of $42 \mathrm{mg}$, with regard to maximum efficiency, preferably after a full night's sleep and in a $120 \mathrm{mg}$ daily dosage has proven to have no statistically significant effect on the outcome (Corponi et al., 2019). It takes 13 to 21 hours for it to be 
absorbed after oral administration. The onset of T-max is about three to four hours from the beginning of the regimen (Vyas et al., 2020; Correll et al., 2020). There is virtually no clearance of lumateperone and its metabolites via the kidneys or urinary tract. Some people who take Lumateperone report a variety of adverse effects, from mild to severe (Krogmann et al., 2019). One of the most often reported adverse effects associated with the FDAapproved dose of $42 \mathrm{mg} /$ day is drowsiness, somnolence, tiredness, and constipation (Correll et al., 2020). Out of a sample of 49 individuals, $17.6 \%$ felt somnolence, $12.7 \%$ reported drowsiness, and $5.3 \%$ expressed tiredness after consuming the current FDA-approved dosage of $42 \mathrm{mg}$. With respect to this group, $63.7 \%$ of individuals had negative outcomes (Correll et al., 2020). Fewer than 5\% of individuals $(n=294)$ had extrapyramidal symptoms (EPS) with lumateperone treatment. Additional research shown to have no statistically significant difference in median weight, which implies that larger weight is not better. Despite this medication not having affinity for many non-target receptors, such as histaminergic and muscarinic receptors, the metabolic end points, such as triglycerides, blood glucose, and prolactin, did not show significant differences from placebo (Correll et al., 2020). A total of 302 patients were assigned to either lumateperone or an already approved antipsychotic medication in a six-week research study. These individuals showed significant improvements in their LDL-cholesterol, triglyceride, and prolactin levels when they switched from another antipsychotic medication to lumateperone (Corponi et al., 2019; Krogmann et al., 2019). However, evidence is currently lacking on the safety and effectiveness of lumateperone. Therefore, several studies on the effects of lumateperone are ongoing, with the aim of evaluating its long-term effects and safety (Calsolaro et al., 2019). Lumateperone is metabolised by the cytochrome P450-3A4 isozyme and interacts 
with inhibitors and stimulators of this isozyme. Lumateperone should not be used in individuals who are taking a medication that activates or inhibits CYP3A4. While this drug also has a sedative effect, it is likely to interact with other sedatives such as alcohol (Correll et al., 2020).

\section{3- Lumateperone and Schizophrenia}

According to the definition, people who have schizophrenia have hallucinations or delusions together with disorganised speech, thoughts, and unpleasant symptoms that have lasted for at least six months (Andreasen and Olsen, 1982; Orrico-Sánchez et al., 2020). Inability to express oneself, considerable loss in speech/communication, lack of motivation, and loss in social drive are all indicators of negative symptoms (American Psychiatric Association, 1994; Leo and Regno, 2000). Schizophrenia afflicted an estimated 20 million individuals globally in 2017 (GBD), with a death rate 2-3 times higher than the normal population (Laursen et al., 2014). Chronic mental illness need lifetime treatment, and one of the pillars of such treatment is antipsychotic medication (Cooper and Gupta, 2020). The first-generation antipsychotics are of little use for movement disorders, Parkinson's disease, and the development of tardive dyskinesia. In contrast to the older generation antipsychotics, which target serotonin and dopamine receptors selectively, newer antipsychotics are associated with metabolic syndrome (Meltzer and McGurk, 1999; Harvey et al., 2016; Remington et al., 2016). In the management of schizophrenia, psychiatrists, nurses, pharmacists, social workers, and other allied healthcare professionals collaborate and contribute to the process. As schizophrenia is a highly complicated condition, it needs a multidisciplinary approach to treatment. Because there is no known cure for schizophrenia, the primary objective of treatment is to relieve the symptoms of the 
disease. While being well and medicated is a key objective, taking care of any seizures or other psychotic episodes is critical (Maroney, 2020). Although schizophrenia's acute symptoms have been well-controlled, its lingering symptoms (which include psychological, behavioural, and social symptoms) need to be dealt with. Thus, just one part of a comprehensive approach to the treatment of schizophrenia patients is medicine. While some forms of psychological and/or psychosocial therapy, as well as other treatment approaches aimed at improving the patient's quality of life, reducing the chances of a relapse, and dealing with residual cognitive and behavioural symptoms that remain after the primary symptoms have been handled, should all be given consideration when treating schizophrenia, other approaches may be even more effective. Without an additional therapy to target other parts of the disease, pharmacological therapies generally fall short of addressing everything related to the condition. As a result, psychologists, social workers, and therapists are involved in the therapeutic process. While the current recommendations of the American Psychiatric Association (APA) stress the importance of personalised pharmacological therapies that consider each patient's unique preferences, clinical response, and side effects, current recommendations from the American Psychiatric Association (APA) argue for the use of customised therapies that incorporate each patient's personal desires, responsiveness, and side effects (Maroney et al., 2020). Patients, as well as the rest of the medical team, should be included in the decision-making process. Schizophrenia without adequate treatment may evolve into a disorder that poses a danger to the patient's health and safety. To get the greatest possible results, healthcare providers must cooperate and communicate efficiently. 


\section{4- Lumateperone and PD}

When dopamine-producing cells die, aggregations of Parkinson disease proteins appear. Dopamine supplements are essential in treating Parkinson's disease. It is hypothesised that, apart from the dopaminergic neurons, other neurotransmitter systems, including as serotonin, acetylcholine, and norepinephrine, are also misfiring in Parkinson disease (Schapira et al., 2017). This explains why $t$ he symptoms of Parkinson's disease are resistant to dopamine-based therapies. Several alternative neurotransmitter systems are targeted by new therapeutic methods.

Intra-Cellular Therapies (which has licence from Bristol-Myers Squibb) is developing a novel orally available medication, Lumateperone, to treat schizophrenia and other neuropsychiatric and neurological disorders. The first in class selective and concurrent serotonin, dopamine, and glutamate modulator, lumateperone, has just arrived on the market. Lumateperone was initially approved in the US for the treatment of schizophrenia in adults in December 2019. Additionally, the drug is in several clinical studies for the treatment of bipolar depression, dementia- and Alzheimer's-related behavioural issues, and insomnia (Vanover et al., 2019).

It is a highly selective antagonist of serotonin 5-HT2A receptors, with an additional property of being an inhibitor of the serotonin transporter (SERT) (Vanover et al., 2019). SERT inhibition causes a stronger antidepressant effect in the presence of the antagonist (Davis et al., 2015). As a partial agonist and an antagonist at dopamine D2 receptors, Lumateperone acts as a presynaptic and postsynaptic modulator of dopamine receptor phosphoprotein D2 (Vanover et al., 2019). Also, it affects the GluN2B glutamate NMDA receptor indirectly via the dopamine D1 receptor (Vanover et al., 2019). 
Levodopa is the most successful treatment for Parkinson's disease, however if the only or the primary symptom is tremor, you should not start using levodopa. Instead, you should choose medicines such as MAO-B inhibitors, amantadine, anticholinergics, or dopamine agonists. A change in levodopa dosage or the addition of a medication that prevents the action of the enzyme monoamine oxidase B (MAO-B) or a catechol-O-methyltransferase inhibitor (usually Entacapone) helps reduce motor fluctuations. Dopamine agonists are most commonly used to treat impulse control disorders. Nonmotor symptoms are limited by a paucity of high-quality positive research, which impacts evidence-based therapy.

Many people with Parkinson's disease require a higher dosage of levodopa every two to three hours, as well as an increase in dosages over time. This is not an indication of drug adverse effects, and does not signal reduced effectiveness. Parkinson disease patients have both a long-lasting and short-lasting loss of their ability to respond to dopaminergic medication, with worsening illness-related pathophysiologic brain changes acting as a second contributing factor (Chou et al., 2018). The brain also loses its ability to store extra dopamine for later use (Chou et al., 2018). Levodopa can be used in combination with a number of other medications. It may be one of these medicines, such as lumateperone.

\subsection{Lumateperone and Dopamine receptor}

Lumateperone also shows functional mesolimbic/mesocortical selectivity in mice, functioning as a postsynaptic antagonist and a presynaptic partial agonist at dopamine D2 receptors in the striatum. Lumateperone activates the signalling protein GSK3, located in dopamine D2 receptor-containing neurons in the prefrontal cortex and nucleus accumbens, 
causing a rise in the phosphorylation of glycogen synthase kinase 3 (GSK3). Dopamine turnover, including the motor side effects and prolactin rise associated with dopamine receptor blockers, can be avoided while still improving antipsychotic effectiveness by administering dopamine receptor blockers that target the mesolimbic/mesocortical and nigrostriatal systems (Correl, 2010).

The affinity of lumateperone $(\mathrm{Ki}=52 \mathrm{nM})$ on dopamine $\mathrm{D} 1$ receptors increases the level of GluN2B (NR2B) phosphorylation of the N-methyl-D-aspartate receptor (NMDAR), and thus augments both AMPA and NMDA channel activity (Davis and Correll, 2016). While schizophrenia sufferers suffer from a deficit in glutamate neurotransmission, which is mediated by NMDA-type receptors, they also have diminished glutamatergic signalling (Lee and Zhou, 2019). Lumateperone regulates the flow of serotonin, dopamine, and glutamate through the brain. The unique pharmacological, pharmacokinetic, and early safety characteristics of Lumateperone point to the medication's ability to serve as a good therapy for schizophrenia. The medicine is swiftly taken into the blood and into the brain, where it then crosses the brain's barrier, and where it is quickly metabolised and then excreted. It has been found to have a significant impact on both positive and negative symptoms of schizophrenia (Davis and Correll, 2016; Lieberman et al., 2016). Saturation occurs at low doses. A different group of receptors is activated at higher dosages, including SERT, D2, D1/GluN2B (glutamate). This allows for its use in a wide range of neuropsychiatric and neurodegenerative diseases, which maximises efficacy while minimising side effects.

Although certain 5-HT2A drugs have shown promise as cognitive enhancers, this is by no means certain. The findings from the early 1990s and the early 2000s suggested that newer 
medications with 5-HT2A activity had better cognitive benefits than previous therapies (Green et al., 1997; Keefe et al., 2004; Woodward et al., 2007). As these studies had methodological faults, they confirmed the findings of the larger trials showing cognitive benefits for each drug (Keefe et al., 2007). Also, the findings show that some antipsychotic medications may have additional receptor properties that cause daytime sleepiness, which decreases cognition instead of improving it (Loebel et al., 2014).

The development of dopamine supersensitivity, an effect of antipsychotic medications, may be mediated by lumateperone. The central nervous system responds by increasing the sensitivity of dopamine receptors in an attempt to overcome the blockage caused by antipsychotic medications, which inhibit dopamine receptors. This supersensitivity is thought to be linked to 5-HT2 receptors, as full expression requires the specific activation of 5-HT2 or 5-HT2A receptors (Charron et al., 2015). These findings show that inhibiting 5-HT2A receptors might assist to avoid the recurrence of the disorder. In this way, even if the treatment of psychotic illnesses is only minimally impacted by the use of serotonin antagonists, these agents may be effective in the treatment of neurodegenerative diseases. Many potential expansions of the use of 5-HT2A-based treatments are present in the diagnosis of schizophrenia. Pimavanserin has just completed a phase 3 trial for dementiarelated psychosis, and it has been licenced for the treatment of Parkinson's psychosis. Likely beneficial in the treatment of Parkinson's disease, Lumateperone may be as well.

In order to fully understand Lumateperone's benefits in the treatment of neurodegenerative disorders, several additional data points will be required. Lumateperone's dopaminergic effects will be investigated. The U.S. Food and Drug Administration (FDA) said that they 
are more willing to consider studies that evaluate dimensional elements of neuropsychiatric diseases, and this might give Lumateperone the opportunity for future study and clinical usage.

\subsection{Lumateprone and Dementia}

Lumateperone binds strongly to serotonin 5-HT2A receptors ( $\mathrm{Ki} 0.54 \mathrm{nM}$ ) and weakly to dopamine D2 receptors ( $\mathrm{Ki} 32 \mathrm{nM}$ ), dopamine D1 receptors ( Ki $52 \mathrm{nM}$ ), and SERT receptors ( $\mathrm{Ki} 62 \mathrm{nM}$ ) (Snyder et al., 2015). Lumateperone also has a moderate affinity for dopamine D4 and adrenergic receptors-1A and -1B (Ki predicted at $100 \mathrm{nM}$ ), but a low affinity for muscarinic and histaminergic receptors (50 percent inhibition at $100 \mathrm{nM}$ ) (Caplyta, 2021). Lumateperone quickly entered the brain in healthy volunteers $(n=16)$, exhibiting long-lasting, dose- and plasma concentration-dependent striatal dopamine D2 receptor occupancy (Davis et al., 2015). Lumateperone $10 \mathrm{mg}$ occupied a high proportion (> 80\% ) of cortical serotonin 5-HT2A receptors and a modest proportion (12\%) of striatal dopamine D2 receptors. Lumateperone $40 \mathrm{mg}$ occupied up to $39 \%$ of striatal dopamine D2 receptors and $33 \%$ of striatal serotonin transporters at their maximum (Davis et al., 2015). In a phase II PET trial (NCT02288845), peak dorsal striatal dopamine D2 receptor occupancy (mean 39\%) occurred at 1 hour post-dose in individuals with schizophrenia $(\mathrm{n}=$ 10) receiving lumateperone $60 \mathrm{mg}$ once daily (Vanover et al., 2019).

Additionally, lumateperone was studied as a possible therapy for behavioural problems in individuals with dementia and other neurological diseases. Ten healthy elderly participants received oral lumateperone (multiple ascending doses up to $30 \mathrm{mg}$ ) or placebo once daily 
for seven days in part 1 of a phase I/II study (ITI-007-200; NCT02078310) (Davis et al., 2014; Intra-Cellular, 2014). Part 2 randomised eight individuals with dementia (including Alzheimer's disease) to receive either oral lumateperone $9 \mathrm{mg}(\mathrm{n}=5)$ or a placebo $(\mathrm{n}=3)$ once daily for seven days. The Hopkins Verbal Learning Test-Revised was used to measure cognitive function. In healthy elderly participants, lumateperone increased verbal learning and memory compared to placebo, but dementia patients treated with lumateperone exhibited improved recognition memory and made fewer false positive mistakes compared to placebo receivers (Davis et al., 2014; Intra-Cellular, 2014).

Finally, and perhaps most significantly, lumateperone's molecular profile is characterised by a high (60-fold) affinity for D2/5-HT2A receptors. The compound's affinity for 5-HT2A receptors is higher than that of numerous antipsychotic drugs studied, including risperidone (10-fold), olanzapine (12-fold), and aripiprazole (0.18-fold). These findings imply that, both preclinically and clinically, increasing the dosage of lumateperone completely engages serotonin 5-HT2A receptors prior to achieving substantial dopamine D2 receptor blockage (and potential motor side effects).

\subsection{Lumateperone and Psychosis}

Hallucinations are a characteristic of late-stage Parkinson disease and a side effect of Parkinson disease treatment, but additional psychotic symptoms are often drug-related. Clozapine and quetiapine have been the most widely researched neuroleptics for the treatment of psychosis in Parkinson disease, owing to the propensity of other neuroleptics 
to exacerbate parkinsonism. Clozapine is a medication that is consistently effective (Eng and Welty, 2010; Goldman et al., 2011). However, because of the danger of agranulocytosis and the necessity for frequent blood testing with clozapine, quetiapine is first given.

\section{5- Conclusions and Clinical perspectives}

Lumateperone market entry provides a revolutionary approach to treat schizophrenia with both a new mechanism of action and a markedly reduced side effects profile. Lumateperone, is a novel second-generation antipsychotic proved to decrease adrenal gland functions with inflammatory degenerative effects on its ultrastructure along with increased expression of IL-6, Caspase-3, and reduced PCNA associated with hypoactive behavior. On the other hand, Quercetin is a potent antioxidant antiinflammatory agent that represents a new prospect for alleviating possible side effects of Lumateperone. Thus, El-Haroun et al., (2021) indicate the robustness of the protective effect of Quercetin against the possible unwanted side effects of Lumateperone. Therefore, they recommend co-administration of Quercetin with Lumateperone as new treatment strategy for schizophrenic patients. This may be an appropriate treatment for patients with treatment-resistant forms of psychotic disorders. A combination of novel antipsychotic drugs and modulating drugs that might improve negative schizophrenic symptoms and cognitive function and thereby social functioning and quality of life. However, further research is needed to determine the safety of this combination using a well-established Schizophrenia animal model. Lumateperone may be of some benefits in treating psychosis, and dementia in neurodegenerative disorders. At least it could be used as an adjunct to the available pharmacological treatment to these disorders to lessen the side effects and 
tolerance by reducing the daily prescribed dose. However, we should consider this with extreme cautious and there is an urgent need for further experimentation of Lumateperone on experimental animal model of neurodegenerative disorders to elucidate any possible drawbacks.

\section{References}

Ahmed M, Malik M, Teselink J, Lanctôt KL, Herrmann N (2019). Current Agents in Development for Treating Behavioral and Psychological Symptoms Associated with Dementia. Drugs Aging. Jul;36(7):589-605. [PubMed]

American Psychiatric Association, (1994). Diagnostic and Statistical Manual of Mental Disorders, Fourth Edition. Washington, DC: American Psychiatric Association.

Andreasen, N.C., Olsen, S., (1982). Negative vs positive schizophrenia definition and validation. Arch Gen Psychiatry. 39, 789-794.

Blair, H.A., (2020). Lumateperone: First Approval. Drugs. 80(4), 417-423.

Calsolaro V, Antognoli R, Okoye C, Monzani F (2019). The Use of Antipsychotic Drugs for Treating Behavioral Symptoms in Alzheimer's Disease. Front Pharmacol. 10:1465. [PMC free article] [PubMed]

Caplyta (lumateperone) (2018). NDA 209500 Multi-disciplinary Review and Evaluation Version date: October 12, Reference ID: 4537404. Multi-Discipline Review (fda.gov) [accessed 3 July 2020]

Charron A, Hage CE, Servonnet A, et al (2015). 5-HT2 receptors modulate the expression of antipsychotic-induced dopamine supersensitivity. Eur Neuropsychopharmacol. Dec;25(12):2381-93.

Chou KL, StacyM, Simuni T, et al (2018). The spectrum of “off" in Parkinson's disease: what have we learned over 40 years? Parkinsonism Relat Disord. 51:9-16. doi:10.1016/j.parkreldis.2018.02.001

Cooper, D., Gupta, V., (2020). Lumateperone. [Updated 2020 Oct 5]. In: StatPearls [Internet]. Treasure Island (FL): StatPearls Publishing; Available from: https://www.ncbi.nlm.nih.gov/books/NBK560844/

Corponi F, Fabbri C, Bitter I, Montgomery S, Vieta E, Kasper S, Pallanti S, Serretti A (2019). Novel antipsychotics specificity profile: A clinically oriented review of lurasidone, 
brexpiprazole, cariprazine and lumateperone. Eur Neuropsychopharmacol. Sep;29(9):971985. [PubMed]

Correll C (2010). From receptor pharmacology to improved outcomes: individualising the selection, dosing, and switching of antipsychotics. Eur Psychiatry. 25:S12-S21.

Correll, C.U., Davis, R.E., Weingart, M., Saillard, J., O'Gorman, C., Kane, J.M., Lieberman, J.A., Tamminga, C.A., Mates, S., Vanover, K.E., (2020). Efficacy and Safety of Lumateperone for Treatment of Schizophrenia: A Randomized Clinical Trial. JAMA Psychiatry. 77(4), 349-358.

Davis R, Saillard J, et al (2014). Safety and tolerability of ITI-007 in patients with dementia: a novel treatment designed to treat behavioral disturbances associated with dementia and related disorders [abstract no. P2-35]. J Prev Alzheimers Dis.;1(3):287-8.

Davis RE, Vanover KE, Zhou Y, et al (2015). ITI-007 demonstrates brain occupancy at serotonin 5-HT2A and dopamine D2 receptors and serotonin transporters using positron emission tomography in healthy volunteers. Psychopharmacology (Berl).232(15):2863-72.

Davis RE, Correll CU (2016). ITI-007 in the treatment of schizophrenia: from novel pharmacology to clinical outcomes. Expert Rev Neurother. 16(6):601-614.

Edinoff A, Wu N, deBoisblanc C, Feltner CO, Norder M, Tzoneva V, Kaye AM, Cornett EM, Kaye AD, Viswanath O, Urits I (2020). Lumateperone for the Treatment of Schizophrenia. Psychopharmacol Bull.14;50(4):32-59. PMID: 33012872; PMCID: PMC7511146.

El-Harun H, Ewida SF, Mohamed W, Bashandy MA (2021). Atypical Antipsychotic Lumateperone effects on the Adrenal Gland with possible beneficial effects of Quercetin $\begin{array}{lllll}\text { co-administration. } & \text { Front. } & \text { Physiol., } & 29 & \text { June }\end{array}$ https://doi.org/10.3389/fphys.2021.674550

Eng ML,Welty TE (2010). Management of hallucinations and psychosis in Parkinson's disease. Am J Geriatr Pharmacother. 8(4):316-330.

Factor SA, McDonald WM, Goldstein FC. The role of neurotransmitters in the development of Parkinson's disease-related psychosis. Eur J Neurol. 2017;24(10):12441254. doi:10.1111/ene.13376

Goldman JG, Vaughan CL, Goetz CG (2011). An update expert opinion on management and research strategies in Parkinson's disease psychosis. Expert Opin Pharmacother.12(13):2009-2024.

Green MF, Marshall BD, Jr., Wirshing WC, et al. Does risperidone improve verbal working memory in treatment-resistant schizophrenia? Am J Psychiatry. 1997 Jun;154(6):799-804. 
Greenwood J, Acharya RB, Marcellus V, Rey JA. Lumateperone: A Novel Antipsychotic for Schizophrenia. Ann Pharmacother. 2021 Jan;55(1):98-104. [PubMed]

Harvey PD, Keefe RS (2001). Studies of cognitive change in patients with schizophrenia following novel antipsychotic treatment. Am J Psychiatry. Feb;158(2):176- 84.

Harvey, R.C., James, A.C., Shields, G.E., 2016. A systematic review and network metaanalysis to assess the relative efficacy of antipsychotics for the treatment of positive and negative symptoms inearly-onset schizophrenia. CNS Drugs. 30(1), 27-39.

Intra-Cellular T. Intra-Cellular Therapies announces additional results from phase I/II clinical trial for ITI-007 in healthy geriatric subjects and patients with dementia [media release]. 24 Nov 2014. http://www.intra cellu larth erapi es.com.

Intra-Cellular Therapies. Caplyta (lumateperone) capsules, for oral use: US prescribing information. 2019. http://www.acces sdata .fda.gov/. Accessed 26 Jul 2021.

Kantrowitz, J.T., 2020. The Potential Role of Lumateperone-Something Borrowed? SomethingNew? JAMA Psychiatry. 77(4), 343-344.

Keefe RS, Seidman LJ, Christensen BK, et al. Comparative effect of atypical and conventional antipsychotic drugs on neurocognition in first-episode psychosis: a randomized, double-blind trial of olanzapine versus low doses of haloperidol. Am J Psychiatry. 2004 Jun;161(6):985-95.

Keefe RS, Bilder RM, Davis SM, et al (2007). Neurocognitive effects of antipsychotic medications in patients with chronic schizophrenia in the CATIE Trial. Arch Gen Psychiatry. Jun;64(6):633-47.

Krogmann A, Peters L, von Hardenberg L, Bödeker K, Nöhles VB, Correll CU. Keeping up with the therapeutic advances in schizophrenia: a review of novel and emerging pharmacological entities. CNS Spectr. 2019 Aug;24(S1):38-69. [PubMed]

Kumar B, Kuhad A, Kuhad A. Lumateperone: a new treatment approach for neuropsychiatric disorders. Drugs Today (Barc). 2018 Dec;54(12):713-719. [PubMed]

Laursen TM, Nordentoft M, Mortensen PB (2014). Excess early mortality in schizophrenia. Annu Rev Clin Psychol. 10:42-48. doi: 10.1146/annurev-clinpsy-032813153657. Epub 2013 Dec 2.

Lee G, Zhou Y (2019). NMDAR hypofunction animal models of schizophrenia. Front Mol Neurosci. July $31 ; 12: 185$.

Leo, R.J., Regno, P.D., 2000. Atypical Antipsychotic Use in the Treatment of Psychosis in Primary Care. Prim Care Companion J Clin Psychiatry. 2(6), 194-204. doi:10.4088/pcc.v02n0601 
Lieberman JA, Davis RE, Correll CU, et al (2016). ITI-007 for the treatment of schizophrenia: A 4-week randomized, double-blind, controlled trial. Biol Psychiatry. 79(12):952-961.

Loebel AD, Siu CO, Cucchiaro JB, et al (2014). Daytime sleepiness associated with lurasidone and quetiapine XR: results from a randomized double-blind, placebo controlled trial in patients with schizophrenia. CNS Spectr.Apr;19(2):197-205.

Maillet A, Krack P, Lhomm.e E, et al. The prominent role of serotonergic degeneration in apathy, anxiety and depression in de novo Parkinson's disease. Brain. 2016;139(Pt 9):2486-2502. doi:10.1093/brain/aww162

Maroney M. An update on current treatment strategies and emerging agents for the management of schizophrenia. Am J Manag Care. 2020 Mar;26(3 Suppl):S55S61. [PubMed]

Mazza M, Marano G, Traversi G, Sani G, Janiri L. Evidence on the New Drug Lumateperone (ITI-007) for Psychiatric and Neurological Disorders. CNS Neurol Disord Drug Targets. 2020;19(4):243-247. [PubMed]

Meltzer, H.Y., McGurk S.R., 1999. The effects of clozapine, risperidone, and olanzapine oncognitive function in schizophrenia. Schizophr Bull. 25(2), 233-55.

Morris R, Martini DN, Madhyastha T, et al. Overview of the cholinergic contribution to gait, balance and falls in Parkinson's disease. Parkinsonism Relat Disord. 2019;63:20-30. doi:10. 1016/j.parkreldis.2019.02.017

Orrico-Sánchez, A., López-Lacort, M., Muñoz-Quiles, C. et al., 2020. Epidemiology of schizophrenia and its management over 8-years period using real-world data in Spain. BMCPsychiatry 20, 149 https://doi.org/10.1186/s12888-020-02538-8

Remington, G., Foussias, G., Fervaha, G., et al., 2016. Treating Negative Symptoms in Schizophrenia: an Update. Curr Treat Options Psychiatry. 3, 133-150. doi:10.1007/s40501016-0075-8

Schapira AHV, Chaudhuri KR, Jenner P. Non-motor features of Parkinson disease. Nat Rev Neurosci. 2017;18(7):435-450. doi:10.1038/nrn.2017.62

Snyder, G., Vanover, K., Zhu, H., et al., 2015. Functional profile of a novel modulator of serotonin, dopamine, and glutamate neurotransmission. Psychopharmacology (Berl). 232(3), 605-621. doi:10.1007/s00213-014-3704-1

Vanover KE, Davis RE, Zhou Y, Ye W, Brašić JR, Gapasin L, Saillard J, Weingart M, Litman RE, Mates S, Wong DF. Dopamine $\mathrm{D}_{2}$ receptor occupancy of lumateperone (ITI007): a Positron Emission Tomography Study in patients with schizophrenia. Neuropsychopharmacology. 2019 Feb;44(3):598-605. [PMC free article] [PubMed] 
Vyas P, Hwang BJ, Brašić JR. An evaluation of lumateperone tosylate for the treatment of schizophrenia. Expert Opin Pharmacother. 2020 Feb;21(2):139-145. [PubMed]

Woodward ND, Purdon SE, Meltzer HY, et al. A meta-analysis of cognitive change with haloperidol in clinical trials of atypical antipsychotics: dose effects and comparison to $\begin{array}{llll}\text { practice } & \text { effects. } & \text { Schizophr } & \text { Res. }\end{array}$ 
